\title{
THE REAL FIELD WITH THE RATIONAL POINTS OF AN ELLIPTIC CURVE
}

\author{
AYHAN GÜNAYDIN AND PHILIPP HIERONYMI
}

\begin{abstract}
We consider the expansion of the real field by a subgroup of a one-dimensional definable group satisfying a certain diophantine condition. The main example is the group of rational points of an elliptic curve over a number field. We prove a completeness result, followed by a quantifier elimination result. Moreover we show that open sets definable in that structure are semialgebraic.
\end{abstract}

\section{INTRODUCTION}

Here we study the model theoretic structure $(\mathbb{R}, \mathcal{E}(\mathbb{Q}))$, where $\mathbb{R}$ is the real field and $\mathcal{E}(\mathbb{Q})$ is the group of rational points of an elliptic curve over a number field. By an elliptic curve over a number field, we mean the collection of solutions $(\alpha, \beta) \in \mathbb{R}^{2}$ of the equation

$$
y^{2}=x^{3}+a x+b \text {. }
$$

with $a, b \in \mathbb{R}$ algebraic over $\mathbb{Q}$ with $4 a^{3}+27 b^{2} \neq 0$, augmented by an element $\mathcal{O}$ of $\mathbb{Q}^{2}$ different than the ones above. Such a collection becomes a onedimensional group definable in the real field (we explain the details of this below in Section 2); and we denote that group as $\mathcal{E}$. For another field $K$, the collection of solutions $(\alpha, \beta) \in K^{2}$ of (1.1) with $\mathcal{O}$ is denoted by $\mathcal{E}(K)$. If $K \subseteq \mathbb{R}$, then $\mathcal{E}(K)$ is a subgroup of $\mathcal{E}$. A rational point of an elliptic curve $\mathcal{E}$ is an element of $\mathcal{E}(\mathbb{Q})$.

We consider $(\mathbb{R}, \mathcal{E}(\mathbb{Q}))$ as a structure in the language $\mathcal{L}_{\mathrm{o}}(P)$ extending the language $\mathcal{L}_{\mathrm{o}}=\{0,1,+, \cdot,<\}$ of ordered rings by a binary relation symbol $P$. Our main result is the following.

Theorem 1.1. Every definable subset of $\mathbb{R}^{s}$ in the structure $(\mathbb{R}, \mathcal{E}(\mathbb{Q}))$ is defined by a boolean combination of formulas of the form

$$
\exists y_{1} \cdots \exists y_{2 n}\left[\bigwedge_{j=0}^{n-1} P\left(y_{2 j+1}, y_{2 j+2}\right) \wedge \phi(x, y)\right],
$$

where $y$ denotes the tuple $\left(y_{1}, \ldots, y_{2 n}\right), x$ is a s-tuple of distinct variables and $\phi(x, y)$ is a quantifier-free $\mathcal{L}_{\mathrm{O}}$-formula.

Date: March 11, 2019.

2000 Mathematics Subject Classification. 03C10, 03C64, 14H52, 11 U09.

The second author was funded by Deutscher Akademischer Austausch Dienst. 
As a by-product of the proof this theorem, we also get an axiomatization of the first order theory of $(\mathbb{R}, \mathcal{E}(\mathbb{Q})$ ) (see Theorem 1.2 below). Moreover using this quantifier elimination, we answer a question from [3] affirmatively; namely we show that an open subset of $\mathbb{R}^{s}$ definable in $(\mathbb{R}, \mathcal{E}(\mathbb{Q}))$ is already definable in the real field $\mathbb{R}$. In particular we have that the open core of $(\mathbb{R}, \mathcal{E}(\mathbb{Q})$ ) is o-minimal (see Section 4 for the definition of the open core of an ordered structure). Also as a consequence of that proof, we were able to remove one of the assumptions from one of the main results of [7].

We handle the structure under consideration in a more general way. Namely we study $(\mathbb{R}, \Gamma)$, where $\Gamma \subseteq \mathbb{R}^{m}$ is a dense subgroup of a group definable in $\mathbb{R}$ of dimension one, satisfying a number theoretic property. Roughly speaking we replace the elliptic curve $\mathcal{E}$ by a one-dimensional definable group and $\mathcal{E}(\mathbb{Q})$ by $\Gamma$. Starting from next paragraph we clarify what we mean by this. Indeed the current paper is not the first attempt to treat such structures. For instance, Zilber studied the real field expanded by the group of roots of unity in [12] and later Belegradek and Zilber generalized the results of that paper to the real field expanded by a finitely generated subgroup of the unit circle in [1. The first author of the current paper studied similar structures in [6] with an approach different than the one in [1].

Let $K$ be a real closed field and let $(\mathbb{A}, \oplus)$ be a group definable in $K$; that is $\mathbb{A} \subseteq K^{m}$ and $\oplus: K^{m} \times K^{m} \rightarrow K^{m}$ are definable in $K$ such that $\oplus \mid(\mathbb{A} \times \mathbb{A})$ is a group operation on $\mathbb{A}$. Suppose also that $\mathbb{A}$ is of dimension one. Then by Proposition 2.5 of [10], we know that $\mathbb{A}$ becomes a topological group, moreover that a subset of $\mathbb{A}$ is dense in $\mathbb{A}$ in that topology if and only if it is dense in the topology induced from $K^{m}$ (this last part is not true for bigger dimensions). In [10, it is also proven that $\mathbb{A}$ is abelian.

For convenience we also assume that $\mathbb{A}$ is definable over $\emptyset$. For another real closed field $L$, we let $(\mathbb{A}(L), \oplus)$ denote the group definable in $L$ by the formulas defining $(\mathbb{A}, \oplus)$ in $K$.

For a tuple $k=\left(k_{1}, \ldots, k_{n}\right)$ of integers define the group character

$$
\chi_{k}: \mathbb{A}^{n} \rightarrow \mathbb{A}, \quad \chi_{k}\left(a_{1}, \ldots, a_{n}\right):=k_{1} a_{1} \oplus \cdots \oplus k_{n} a_{n} .
$$

For such $k$, let $T_{k}$ denote the kernel of $\chi_{k}$. Also if $G$ is a subgroup of $\mathbb{A}$ and $n>0$, then we put

$$
\begin{gathered}
n G:=\{n g: g \in G\}, \\
G[n]:=\{g \in G: n g=0\}, \quad \text { the } n \text {-torsion subgroup of } G \text {. }
\end{gathered}
$$

We say that a subgroup $G$ of $\mathbb{A}$ has the Mordell-Lang property if for every algebraic set $V \subseteq\left(K^{m}\right)^{n}$ over $\mathbb{Q}$, there are $g_{1}, \ldots, g_{t} \in G^{n}$ and tuples $k_{1}, \ldots, k_{t} \in \mathbb{Z}^{n}$ such that

$$
V \cap G^{n}=\bigcup_{i=1}^{t} g_{i} \oplus\left(T_{k_{i}} \cap G^{n}\right) .
$$


The reason for calling this property with this name is that it is the conclusion of a conjecture of Lang generalizing a conjecture of Mordell for abelian varieties. We refer the reader to [9] for the precise statement of the conjecture and its history.

In the rest of this introduction let $\mathbb{A}$ be $\mathbb{A}(\mathbb{R}) \subseteq \mathbb{R}^{m}$. It is well-known that if $\mathbb{A}$ is connected, then it becomes a real Lie group of dimension one, and hence it is homeomorphic to either the additive group real numbers or the circle group $\mathbb{S}$. Then it is easy to see that $\mathbb{A}$ either is torsion free or has $p$ elements in $\mathbb{A}[p]$ for every prime $p$. Even when $\mathbb{A}$ is not connected, we still have a good control over the torsion elements of $\mathbb{A}$ as the connected component of $\mathbb{A}$ is of finite index in $\mathbb{A}$. Now let $\Gamma$ be a dense subgroup of $\mathbb{A}$ with the Mordell-Lang property such that $|\Gamma / n \Gamma|$ is finite for every $n>0$. Since $n \Gamma$ is the image of $\Gamma$ under a continuous map, $n \Gamma$ is dense in $\Gamma$.

Fix $n>0$ and a tuple of distinct indeterminates $X=\left(X_{1}, \ldots, X_{m n}\right)$. In what follows we identify $\mathbb{R}^{m n}$ with $\left(\mathbb{R}^{m}\right)^{n}$ and for $\gamma_{1}, \ldots, \gamma_{n} \in \Gamma$ and a polynomial $p(X) \in \mathbb{Q}[X], p\left(\gamma_{1}, \ldots, \gamma_{n}\right)$ means

$$
p\left(\pi_{1}\left(\gamma_{1}\right), \pi_{2}\left(\gamma_{1}\right), \ldots, \pi_{m}\left(\gamma_{1}\right), \ldots, \pi_{1}\left(\gamma_{n}\right), \ldots, \pi_{m}\left(\gamma_{n}\right)\right),
$$

where $\pi_{1}, \ldots, \pi_{m}$ are the standard projections of $\mathbb{R}^{m}$ onto $\mathbb{R}$. Note that as $\Gamma$ is included in a 1-dimensional definable subset of $\mathbb{R}$, there is $i \in\{1, \ldots, m\}$ such that for $j=1, \ldots, m$ every element of $\pi_{j}(\Gamma)$ is definable over $\pi_{i}(\Gamma)$. For our purposes it is harmless to assume that $i=1$ and we write $\pi$ instead of $\pi_{1}$.

Also for a polynomial $p$ as above we put

$$
V(p):=\left\{\alpha \in \mathbb{R}^{m n}: p(\alpha)=0\right\},
$$

the zero set of $p(X)$ (in $\mathbb{R})$.

Let $\mathcal{L}_{\mathrm{o}}(P)$ be the extension of $\mathcal{L}_{\mathrm{o}}$ by an $m$-ary relation symbol $P$. Moreover let $\mathcal{L}_{\mathrm{o}}(\Gamma)$ be the language $\mathcal{L}_{\mathrm{o}}$ augmented by a constant symbol for each element of $\pi(\Gamma)$ and similarly let $\mathcal{L}_{\mathrm{o}}(P ; \Gamma)$ be the language $\mathcal{L}_{\mathrm{o}}(P)$ extended by by adding a name for each element of $\pi(\Gamma)$. For a polynomial $p(X) \in$ $\mathbb{Q}[X]$ fix finite sets $\left\{k_{1}, \ldots, k_{t}\right\} \subseteq \mathbb{Z}^{n}$ and $\left\{\gamma_{1}, \ldots, \gamma_{t}\right\} \subseteq \Gamma^{n}$ such that

$$
V(p) \cap \Gamma^{n}=\bigcup_{i=1}^{t} \gamma_{i} \oplus\left(T_{k_{i}} \cap \Gamma^{n}\right) .
$$

Then the Mordell-Lang axiom for $p$ is the $\mathcal{L}_{\mathrm{o}}(P ; \Gamma)$-sentence

$$
\begin{gathered}
\forall x_{1} \ldots \forall x_{m n}\left[\bigwedge_{j=0}^{n-1} P\left(x_{j m+1}, \ldots, x_{j m+m}\right) \wedge p\left(x_{1}, \ldots, x_{m n}\right)=0 \leftrightarrow\right. \\
\left.\bigvee_{i=1}^{t} \chi_{k_{i}}\left(\left(x_{1}, \ldots, x_{m}\right), \ldots,\left(x_{m n-m+1}, \ldots, x_{m n}\right)\right)=\gamma_{i}\right] .
\end{gathered}
$$

(To be precise, the last part of the formula above is short for the obvious $\mathcal{L}_{\mathrm{o}}(\Gamma)$-formula, for example $\gamma_{i}$ is short for $\left(\pi_{1}\left(\gamma_{i}\right), \ldots, \pi_{m}\left(\gamma_{i}\right)\right)$.) 
We construe $\left(\mathbb{R}, \Gamma,\left(\pi(\gamma)_{\gamma \in \Gamma}\right)\right)$ as an $\mathcal{L}_{\mathrm{o}}(P ; \Gamma)$-structure, and axiomatize its first order theory as follows.

Theorem 1.2. Let $K$ be a real closed field and $G$ a dense subgroup of $\mathbb{A}(K)$ with a group morphism $\gamma \mapsto \gamma^{\prime}: \Gamma \rightarrow G$. Let $\Gamma^{\prime}$ be the image of $\Gamma$ under this map. Then

if and only if

$$
\left(K, G,\left(\pi\left(\gamma^{\prime}\right)\right)_{\gamma \in \Gamma}\right) \equiv\left(\mathbb{R}, \Gamma,(\pi(\gamma))_{\gamma \in \Gamma}\right)
$$

- $\left(K,\left(\pi\left(\gamma^{\prime}\right)\right)_{\gamma \in \Gamma}\right) \equiv\left(\mathbb{R},\left(\pi(\gamma)_{\gamma \in \Gamma}\right)\right)$,

- $\Gamma^{\prime}$ is a pure subgroup of $G$,

- for every $n>0$, we have that $G[n]=\Gamma[n]$ and $|G / n G|=|\Gamma / n \Gamma|$,

- $\left(K, G,\left(\pi\left(\gamma^{\prime}\right)\right)_{\gamma \in \Gamma}\right)$ satisfies the Mordell-Lang axioms for $\Gamma$.

Conventions and notations. Above and in the rest of the paper $m, n$ always denote natural numbers. Also as usual 'definable' means 'definable with parameters' and when we want to make the language and the parameters explicit we write $\mathcal{L}$ - $B$-definable to mean definable (in the appropriate structure) in the language $\mathcal{L}$ using parameters from the set $B$.

The real closure of an ordered field $K$ is denoted by $K^{\mathrm{rc}}$.

We denote the graph of a function $f: X \rightarrow Y$ by $\operatorname{gr}(f)$.

Acknowledgements. We would like to thank L. van den Dries, C. Ealy, C. Miller, S. Starchenko and B. Zilber for useful discussions on the topic. Also we would like to thank The Fields Institute for hospitality during the 'Thematic Program on O-minimal Structures and Real Analytic Geometry'; most of this paper was written during that period.

\section{The Mordell-LANG PROPERTy}

Throughout this section $K$ is a real closed field and $\mathbb{A} \subseteq K^{m}$ is an $\mathcal{L}_{\mathrm{O}^{-}}$ definable group over $\mathbb{Q}$ of dimension one, with the group operation $\oplus$. (The assumption that $\mathbb{A}$ is defined over $\mathbb{Q}$ is for convenience.) Let $G$ be a subgroup of $\mathbb{A}$. We construe $(K, G)$ as an $\mathcal{L}_{\mathrm{o}}(P)$-structure in the obvious way.

For $k=\left(k_{1}, \ldots, k_{n}\right) \in \mathbb{Z}^{n}$ and $m \in \mathbb{Z}$, define

$$
D_{k, m}:=\chi_{k}^{-1}(m G) \cap G^{n} \text {. }
$$

Note that $D_{k, m}$ is a subset of $G^{n}$ definable in $\mathcal{L}_{\mathrm{o}}(P)$ and that $(m G)^{n} \subseteq D_{k, m}$. Hence whenever $m G$ is of finite index in $G$ we have that $D_{k, m}$ is of finite index in $G^{n}$ and moreover that both $D_{k, m}$ and $G^{n} \backslash D_{k, m}$ are finite unions of cosets (in $G^{n}$ ) of $(m G)^{n}$. Using the fact that the collection $\left\{(m G)^{n}: m>0\right\}$ is a distributive lattice of subgroups of $G^{n}$, we get the following consequence.

Lemma 2.1. Let $n>0, k_{1}, \ldots, k_{s} \in \mathbb{Z}^{n}$ and $m_{1}, \ldots, m_{t}>0$. Suppose that $\left|G / m_{j} G\right|$ is finite for $j=1, \ldots, t$. Then every boolean combination (in $\left.G^{n}\right)$ of cosets of $D_{k_{i}, m_{j}}$ in $G^{n}$ with $i \in\{1, \ldots, s\}$ and $j \in\{1, \ldots, t\}$ is a finite union of cosets of $(M G)^{n}$, where $M$ is the lowest common multiple of $m_{1}, \ldots, m_{t}$. 
Remark. Note that $M$ in the lemma depends only on $m_{1}, \ldots, m_{t}$ and not on $G$ or $k_{1}, \ldots, k_{s}$.

In the rest of this section $L$ is a subfield of $K$, and $L^{\text {rc }}$ denotes the real closure of $L$ in $K$. For a subset $S$ of $G, L(S)$ denotes the subfield

$$
L\left(\pi_{1}(S) \cup \cdots \cup \pi_{m}(S)\right)
$$

of $K$. Similarly $L[S]:=L\left[\pi_{1}(S) \cup \cdots \cup \pi_{m}(S)\right]$. As mentioned in the introduction there is $i \in\{1, \ldots, m\}$ such that for $j=1, \ldots, m$ every element of $\pi_{j}(G)$ is definable over $\pi_{i}(G)$; for convenience we assume that $i=1$ and most of the time write $\pi$ instead of $z$. It is worth noting then that $E(G)^{\mathrm{rc}}=E(\pi(G))^{\mathrm{rc}}$.

Also $X=\left(X_{1}, \ldots, X_{m n}\right)$ denotes a tuple of distinct indeterminates.

Definition 2.2. We say that $G$ has the Mordell-Lang property over $L$ if for every polynomial $p(X) \in L[X]$, the set

$$
V(p) \cap G^{n}=\left\{\left(g_{1}, \ldots, g_{n}\right) \in G^{n}: p\left(g_{1}, \ldots, g_{n}\right)=0\right\}
$$

is a finite union of cosets of $T_{k} \cap G^{n}$ in $G^{n}$ for various $k \in \mathbb{Z}^{n}$.

We are aiming to show that if $G$ has the Mordell-Lang property over $\mathbb{Q}$, then it has the Mordell-Lang property over $K$.

Lemma 2.3. Let $L$ contain $\mathbb{Q}(G)$ and suppose that $G$ has the Mordell-Lang property over $L$. Then $G$ has the Mordell-Lang property over $L^{\mathrm{rc}}$.

Proof. Let $\alpha \in K$ be algebraic over $L$ of degree $d>1$. It suffices to show that $G$ has the Mordell-Lang property over $L(\alpha)$. Take a polynomial $p(X) \in$ $L[\alpha][X]$. Write

$$
p(X)=p_{0}(X)+p_{1}(X) \alpha+\cdots+p_{d-1}(X) \alpha^{d-1},
$$

where $p_{i}(X) \in L[X]$ for $i=0,1, \ldots, d-1$. Then for $g=\left(g_{1}, \ldots, g_{n}\right) \in G^{n}$

$$
p(g)=0 \Longleftrightarrow p_{i}(g)=0 \text { for each } i \in\{0,1, \ldots, d-1\} .
$$

Therefore

$$
V(p) \cap G^{n}=\bigcap_{i=0}^{d-1} V\left(p_{i}\right) \cap G^{n}=V\left(p_{1}^{2}+\cdots+p_{d-1}^{2}\right) \cap G^{n} .
$$

By the Mordell-Lang property over $L$, we know that $V\left(p_{1}^{2}+\cdots+p_{d-1}^{2}\right) \cap G^{n}$ is a finite union of cosets of kernels of $\chi_{k}$ in $G^{n}$, thus so is $V(p) \cap G^{n}$.

We need the following piece of notation in the next step: For $s \in \mathbb{N}$ and $i=(i(1), \ldots, i(s)) \in \mathbb{N}^{s},|i|$ denotes $i(1)+\cdots+i(s)$, and for a tuple $Y=$ $\left(Y_{1}, \ldots, Y_{s}\right)$ of distinct indeterminates, $Y^{i}$ is the monomial

$$
Y_{1}^{i(1)} Y_{2}^{i(2)} \cdots Y_{s}^{i(s)} \text {. }
$$

Likewise for $\alpha=\left(\alpha_{1}, \ldots, \alpha_{s}\right) \in K^{s}, \alpha^{i}$ means $\alpha_{1}^{i(1)} \alpha_{2}^{i(2)} \cdots \alpha_{n}^{i(s)}$. 
Lemma 2.4. Let $G$ have the Mordell-Lang property over $\mathbb{Q}$. Then $G$ has the Mordell-Lang property over $\mathbb{Q}(G)$.

Proof. Take a polynomial $p(X) \in \mathbb{Q}[G][X]$ of degree $d$. Write

$$
p(X)=\sum_{|i| \leq d} \sum_{j} a_{i, j} g^{j} X^{i}
$$

where $i$ and $j$ run through elements of $\mathbb{N}^{m n}$ and $\mathbb{N}^{m t}$ respectively, $a_{i, j} \in \mathbb{Q}$, and $g=\left(g_{1}, \ldots, g_{t}\right) \in G^{t}$.

Let $Y=\left(Y_{1}, \ldots, Y_{m t}\right)$ be a tuple of indeterminates different than $X$ and put $q(X, Y)=\sum_{|i| \leq d} \sum_{j} a_{i, j} X^{i} Y^{j} \in \mathbb{Q}[X, Y]$. For $g^{*} \in G^{n}$ we have

$$
p\left(g^{*}\right)=0 \Longleftrightarrow q\left(g^{*}, g\right)=0 .
$$

Now the result follows since $G$ has the Mordell-Lang property over $\mathbb{Q}$.

Proposition 2.5. Let $G$ have the Mordell-Lang property over $\mathbb{Q}$. Then $G$ has the Mordell-Lang property over $K$.

Proof. Let $E \subseteq K$ be a finitely generated extension of $\mathbb{Q}(G)^{\mathrm{rc}}$, and take a transcendence basis $\alpha=\left(\alpha_{1}, \ldots, \alpha_{t}\right)$ of $E$ over $\mathbb{Q}(G)^{\mathrm{rc}}$.

Take a polynomial $p(X) \in E[X]$, and write

$$
p(X)=\sum_{i} p_{i}(X) \alpha^{i},
$$

where $i=(i(1), \ldots, i(t))$ runs through elements of $\mathbb{N}^{t}$ such that $|i| \leq s$ for some $s \in \mathbb{N}$ and $p_{i}(X) \in \mathbb{Q}(G)^{\mathrm{rc}}[X]$.

Now it is easy to see that for $g \in G^{n}$

$$
p(g)=0 \Longleftrightarrow p_{i}(g)=0 \text { for each } i .
$$

Hence $V(p) \cap G^{n}$ is of the desired form since $G$ has the Mordell-Lang property over $\mathbb{Q}(G)^{\mathrm{rc}}$ by the previous two lemmas.

From now on we assume that $G$ has the Mordell-Lang property over $\mathbb{Q}$. As a consequence of the proposition above it is harmless to simply say that $G$ has the Mordell-Lang property.

Let $H$ be a subgroup of $G$ and let $g, g_{1}, \ldots, g_{n}$ be elements of $G$. Put

$$
H_{G}\langle g\rangle:=\{g \in G: \text { there are } h \in H, k \in \mathbb{Z}, n>0 \text { such that } n g=h \oplus k g\} .
$$

We say that $g$ is algebraic over $L$ if $g \in\left(L^{\mathrm{rc}}\right)^{m}$, and that $g_{1}, \ldots, g_{n}$ are algebraically dependent over $L$ of there is a polynomial $p(X) \in L[X]$ such that $p\left(g_{1}, \ldots, g_{n}\right)=0$. Also we say that $g_{1}, \ldots, g_{n}$ are linearly dependent over $H$ if there is $k=\left(k_{1}, \ldots, k_{n}\right) \in \mathbb{Z}^{n}$ such that $\chi_{k}\left(g_{1}, \ldots, g_{n}\right) \in H$.

Now let $p(X) \in K[X]$. Say

$$
V(p) \cap G^{n}=g_{1} \oplus T_{1} \cup \cdots \cup g_{s} \oplus T_{s}
$$


where $g_{1}, \ldots, g_{s} \in G^{n}$ and $T_{i}=T_{k_{i}}$ with $k_{i} \in \mathbb{Z}^{n}$ for $i=1, \ldots, s$. Then we say that $p$ has the same Mordell-Lang solutions in $H$ as in $G$ if $g_{1}, \ldots, g_{s}$ can be chosen form $H^{n}$ and

$$
V(p) \cap H=g_{1} \oplus\left(T_{1} \cap H^{n}\right) \cup \cdots \cup g_{s} \oplus\left(T_{s} \cap H^{n}\right) .
$$

We say $G$ and $H$ have the same Mordell-Lang solutions over $L$ if every polynomial $p(X) \in L[X]$ has the same Mordell-Lang solutions in $H$ as in $G$.

The next result is an analogue of Lemma 5.12 in [4].

Lemma 2.6. Let $H$ be a subgroup of $G$ and $g, g_{1}, \ldots, g_{s} \in G$. Suppose that $G$ and $H$ have the same Mordell-Lang solutions over $L$. Then we have:

(1) If $g$ is algebraic over $L(H)$, then $d g \in H$ for some $d>1$.

(2) If $g_{1}, \ldots g_{s}$ are algebraically dependent over $L(H)$, then they are linearly dependent over $H$.

Proof. We just prove (1), and (2) can be proven using similar arguments. Let $X=\left(X_{1}, \ldots, X_{m}\right)$ and $Y=\left(Y_{1}, \ldots, Y_{m t}\right)$ be tuples of distinct indeterminates, and take a polynomial $P(X, Y) \in L[X, Y]$ and $h \in H^{t}$ such that $p(g, h)=0$. Then by the assumption there exist $d \in \mathbb{Z}$ and $k \in \mathbb{Z}^{t}$ such that $(g, h) \in h^{*} \oplus\left(T_{d, k} \cap G^{1+t}\right)$ for some $h^{*} \in H^{1+t}$. Now it is easy to see that $d g \in H$.

2.1. Elliptic curves. As mentioned in the introduction our main example of a group with the Mordell-Lang property is the group of rational points of an elliptic curve over $\mathbb{Q}^{\mathrm{rc}}$. Here we briefly explain how such an object fits into our framework. First of all, in contrast with the introduction, here we use $\mathcal{E}$ for the complex points of an elliptic curve defined by parameters $a, b \in \mathbb{R}$ that are algebraic over $\mathbb{Q}$ with $4 a^{3}+27 b^{2} \neq 0$, and $\mathcal{E}(\mathbb{R})$ for the set of real points of the same elliptic curve. It is more natural to work in the complex projective plane $\mathbb{P}^{2}$. Then $\mathcal{E}$ consists of $(\alpha: \beta: \gamma) \in \mathbb{P}^{2}$ such that

$$
\beta^{2} \gamma=\alpha^{3}+a \alpha \gamma^{2}+b \gamma^{3}
$$

Hence besides the points $(\alpha: \beta: 1)$, the elliptic curve $\mathcal{E}$ contains also $\mathcal{O}:=(0: 1: 0)$, and $\mathcal{E}$ becomes a group with the identity element $\mathcal{O}$, moreover the group operation is given by rational functions over $\mathbb{Q}$ (see for instance III.2.3 in [11]).

This way $\mathcal{E}$ becomes a complex Lie group and it is analytically isomorphic to an abelian group $\mathbb{C} / \Lambda$ where $\Lambda \subseteq \mathbb{C}$ is a lattice; which can be taken to be $\mathbb{Z}+\mathbb{Z} \theta \sqrt{-1}$ for some $\theta \in \mathbb{R}$ since $\mathcal{E}$ is defined over $\mathbb{Q}^{\text {rc }}$ (see VI.5.5 and VI.6.7(d) in [11]). This isomorphism uses the Weierstrass elliptic function $\wp$ attached to $\Lambda$, namely

$$
z+\Lambda \mapsto \begin{cases}\left(\wp(z): \wp^{\prime}(z): 1\right) & \text { if } z \notin \Lambda, \\ \mathcal{O} & \text { otherwise. }\end{cases}
$$

The endomorphism ring of $\mathcal{E}$ (set of all self-morphisms taking $\mathcal{O}$ to itself) is either $\mathbb{Z}$ or $\mathbb{Z}[\tau]$ for some $\tau \in \mathbb{C}$ with $\tau^{2}$ is a negative integer. In the second case, we say $\mathcal{E}$ has complex multiplication by $\tau$. 
Next we explain why $\mathcal{E}(\mathbb{Q})$ has the Mordell-Lang property. We use the following special case of Falting's Theorem (see [5]).

Theorem 2.7. Let $\mathcal{E}$ be an elliptic curve over $\mathbb{Q}^{\mathrm{rc}}$ and $\Gamma$ a finitely generated subgroup of $\mathcal{E}$. Then for every algebraic subset $V$ of $\mathcal{E}^{n}$, the set $\mathcal{E}^{n} \cap \Gamma^{n}$ is a finite union of cosets of subgroups $P \cap \Gamma^{n}$ of $\Gamma^{n}$, where $P$ is an algebraic subgroup of $\mathcal{E}^{n}$.

By the Mordell-Weil Theorem, $\mathcal{E}(\mathbb{Q})$ is indeed a finitely generated subgroup of the elliptic curve $\mathcal{E}$. In the case that $\mathcal{E}$ does not have complex multiplication, all algebraic subgroups of $\mathcal{E}^{n}$ are given as the kernels of maps of the form

$$
\left(x_{1}, \ldots, x_{n}\right) \mapsto k_{1} x_{1} \oplus \cdots \oplus k_{n} x_{n}: \mathcal{E}^{n} \rightarrow \mathcal{E}
$$

where $k_{i} \in \mathbb{Z}$ for $i=1, \ldots, n$. Hence $\mathcal{E}(\mathbb{Q})$ has the Mordell-Lang property by Theorem 2.7.

Now consider an elliptic curve $\mathcal{E}$ with complex multiplication by $\tau$. In this case, it is a bit more complicated to show that $\mathcal{E}(\mathbb{Q})$ has the Mordell-Lang property, because an algebraic subgroup of $\mathcal{E}^{n}$ is of the kernel of a map of the form

$$
\left(x_{1}, \ldots, x_{n}\right) \mapsto \bigoplus_{i=1}^{n}\left(a_{i}+b_{i} \tau\right) x_{i},
$$

with $a_{i}, b_{i} \in \mathbb{Z}$. But the next Lemma implies that cosets of an intersection of such an algebraic subgroup of form 2.1 with $\mathcal{E}(\mathbb{Q})^{n}$ is a finite union of cosets of the intersection of $\mathcal{E}(\mathbb{Q})^{n}$ with subgroups defined by equations $\bigoplus_{i=1}^{n} a_{i} x_{i}=0$, with $a_{i} \in \mathbb{Z}$.

Lemma 2.8. The intersection of $\mathcal{E}(\mathbb{R})$ with its image under $\tau$ is finite.

Proof. In this case $\mathcal{E}$ is isomorphic to $\mathbb{C} / \Lambda$, where $\Lambda=\mathbb{Z}+\mathbb{Z} \tau$. Since $\mathcal{E}$ is defined over $\mathbb{Q}$, the series expansions of $\wp$ and $\wp^{\prime}$ have only real coefficients. Hence $\wp$ and $\wp^{\prime}$ map equivalence classes of elements of the real axis to elements of the real axis. In particular, $\left(\wp, \wp^{\prime}\right)$ maps the set $\{t+\Lambda: t \in$ $\mathbb{R} \cap[0,1)\}$ into $\mathcal{E}(\mathbb{R})$. Let $S$ be the inverse image of $\mathcal{E}(\mathbb{R})$ under $\left(\wp, \wp^{\prime}\right)$. Because the real points of $\mathcal{E}$ form a one dimensional subgroup of $\mathcal{E}$ which has at most two connect components, $S$ is either

$$
\{t+\Lambda: t \in \mathbb{R} \cap[0,1)\} \text { or }\left\{t+\frac{s}{2} \cdot \tau+\Lambda: t \in \mathbb{R} \cap[0,1), s \in\{0,1\}\right\} .
$$

In other words, $S$ is a finite union of lines parallel to the real axis. Since on $\mathbb{C} / \Lambda$ the endomorphism of $\mathcal{E}$ corresponding to $\tau$ is just multiplication by the complex number $\tau$, the set $\tau S$ is a finite union of lines parallel to the imaginary axis. Hence the intersection of $S$ and $\tau S$ is finite and so is the intersection of $\mathcal{E}(\mathbb{R})$ and its image under $\tau$.

For our purposes it is more convenient to replace $(0: 1: 0)$ by a point of $\mathbb{Q}^{2}$ and carry the group structure to this subset of $\mathbb{C}^{2}$; we still denote that group 
by $\mathcal{E}$. Then $\mathcal{E}(\mathbb{R}):=\mathcal{E} \cap \mathbb{R}^{2}$ becomes a subgroup of $\mathcal{E}$ definable in $\mathbb{R}$ over $\mathbb{Q}$, moreover it is of dimension 1 . Finally if $\mathcal{E}(\mathbb{Q})$ is infinite, $\mathcal{E}(\mathbb{Q}):=\mathcal{E} \cap \mathbb{Q}^{2}$ becomes a dense subgroup of $\mathcal{E}(\mathbb{R})$, because of compactness of $\mathcal{E}$. Note that in the case $\mathcal{E}(\mathbb{Q})$ is finite, Theorem 1.1 holds trivially.

2.2. Smallness revisited. Here we use the definition of smallness from [2], rather than the one in [4]. The reason for this is that in [4] smallness is defined only for subset of $K$ and we are aiming to show that $\Gamma \subseteq K^{m}$ is small in the field $K$.

Recall that for a positive integer $k$, a $k$-valued map, denoted as $f: X \stackrel{k}{\longrightarrow} Y$, is a map from $X$ to $\mathcal{P}(Y)$ such that $|f(x)| \leq k$ for every $x \in X$; and such a map is definable in a given structure if its graph

$$
\{(x, y) \in X \times Y: y \in f(x)\}
$$

is definable in that structure.

Now a subset $X$ of $K^{s}$ is called large if there is a $k$-valued map $f: K^{s n} \stackrel{k}{\longrightarrow} K$ definable in the field $K$ such that $f\left(X^{n}\right)=K$; otherwise we say that $X$ is small. Note that smallness is an elementary property of the pair $(K, X)$ construed as a structure in the language of ordered rings expanded by an $s$-ary relation symbol. It is also easy to see that a set $X \subseteq K^{s}$ is large if and only if there is a usual function $g: K^{s n^{\prime}} \rightarrow K$ definable in the field $K$ such that $g\left(X^{n^{\prime}}\right)=K$ (This is still true if $K$ is replaced by an algebraically closed field, but requires more work).

We first prove a result that has been neglected in the writing of $[2]^{1}$. This result must be known by many people, but we could not find a reference for it anywhere. So we include a proof as well.

Lemma 2.9. Let $f: K^{s} \rightarrow K$ be definable in the field $K$, and let $C$ be the algebraic closure, $K(\sqrt{-1})$, of $K$. Then there is a $k$-valued function $\tilde{f}: C^{s} \stackrel{k}{\longrightarrow} C$ definable in the field $C$ such that $f(\alpha) \in \tilde{f}(\alpha)$ for each $\alpha \in K^{s}$.

Proof. Let $X \subseteq K^{s+1}$ be the graph of $f$. Write $X$ as $\bigcup_{i=1}^{t} U_{i} \cap Z_{i}$ where each $U_{i}$ is a nonempty open subset of $K^{s+1}$ defined by polynomial inequalities and each $Z_{i}$ is an intersection of zero sets in $K$ of polynomials. Since for each $\alpha \in K^{s}, X(\alpha)=\{f(\alpha)\}$ and in particular is of dimension zero, $X$ contains no open set. This means that none of the $Z_{i}$ 's is all of $K^{s+1}$.

Let $\tilde{Z}_{i} \subseteq C^{s+1}$ be the zero set (in $C^{s+1}$ ) of the polynomials defining $Z_{i}$. Note that $\tilde{Z}_{i} \cap K^{s+1}=Z_{i}$.

There is a definable subset $V_{i}$ of $C^{s}$ of dimension less than $s$ such that for every $\alpha \in C^{s} \backslash V_{i}$, the fiber $\tilde{Z}_{i}(\alpha)$ is a finite set. Indeed if there were a definable subset of $C^{s}$ of dimension $s$ with 1-dimensional fibers of $Z_{i}$ above it, then $\tilde{Z}_{i}$ would be of dimension $s+1$, and this could only happen if $\tilde{Z}_{i}$ were all of $C^{s+1}$. Moreover for $i=1, \ldots, t$ there is $N_{i} \in \mathbb{N}$ such that $\left|\tilde{Z}_{i}(\alpha)\right| \leq N_{i}$

\footnotetext{
${ }^{1}$ We would like to thank C. Ealy for helping with this result
} 
for every $\alpha \in C^{s} \backslash V_{i}$. Let $D_{i} \subseteq K$ be the projection of $U_{i} \cap Z_{i}$ to the first $s$ coordinates. We claim that $D_{i}$ is contained in $C^{s} \backslash V_{i}$. Suppose not and take $\alpha \in V_{i} \cap D_{i}$. As $\alpha \in D_{i}$, we have $U_{i} \cap Z_{i}(\alpha)$ is a singleton, namely $\{f(\alpha)\}$. On the other hand, since $\alpha \in V_{i}, \tilde{Z}_{i}(\alpha)$ is an infinite subset, and in particular, an infinite subset of $\{\alpha\} \times C \subseteq C^{s+1}$ that is definable in the field $C$. Since $C$ is an algebraically closed field, $\tilde{Z}_{i}(\alpha)$ is a cofinite subset of $\{\alpha\} \times C$. But that would mean that $U_{i} \cap \tilde{Z}_{i}(\alpha)=U_{i} \cap Z_{i}(\alpha)$ would be an infinite set, providing the contradiction that proves the claim.

Now define $\tilde{f}$ as follows: For $\alpha \in C^{s} \backslash V_{i}$, let $\tilde{f}_{i}(\alpha)=Z_{i}(\alpha)$. Then put $\tilde{f}:=\bigcup_{i=1}^{m} \tilde{f}_{i}$. Note that on $D_{i}, \tilde{f}_{i}(x)$ contains $f(x)$. The union of the $D_{i}$ 's is $K^{s}$, so the lemma follows.

Lemma 2.10. The group $\Gamma$ is small in $K$.

Proof. Construe $(K, \Gamma)$ as a structure in the language $\mathcal{L}_{\mathrm{o}}(P)$, and take a proper elementary extension $\left(K^{*}, \Gamma^{*}\right)$. It suffices to show that $\Gamma^{*}$ is small in $K^{*}$. By the previous lemma it is enough to prove that $\mathbb{Q}\left(\Gamma^{*}\right)$ is small in the algebraic closure $K^{*}(\sqrt{-1})$ of $K^{*}$. Using [8] it is enough to show that $\mathbb{Q}\left(\Gamma^{*}, \sqrt{-1}\right) \neq K^{*}(\sqrt{-1})$. This means we need to show that $\mathbb{Q}\left(\Gamma^{*}\right) \neq K^{*}$.

Take a subset $B$ of $\Gamma^{*}$ that is linearly independent over $\Gamma$ and is maximal with respect to this property. Clearly $B \neq \emptyset$. By Lemma 2.6 we have that $B$ is algebraically independent over $\mathbb{Q}(\Gamma)$, and hence $\mathbb{Q}(\Gamma, B)$ is a purely transcendental extension of $\mathbb{Q}(\Gamma)$. Then $\mathbb{Q}\left(\Gamma^{*}\right)$ cannot be real closed, as for every $\gamma^{*} \in \Gamma^{*}$, there is a positive integer $d$ such that $\left(\gamma^{*}\right)^{d} \in \mathbb{Q}(\Gamma, B)$. Hence $\mathbb{Q}\left(\Gamma^{*}\right) \neq K^{*}$.

A consequence of smallness is the following.

Corollary 2.11. Let $f: K^{m n} \rightarrow K$ be definable in the field $K$. Then $K \backslash f\left(\Gamma^{n}\right)$ is dense in $K$.

Proof. Assume that a nonempty interval $I$ of $K$ is contained in $f\left(\Gamma^{n}\right)$. Take a function $g: K \rightarrow K$ definable in the ordered field $K$ that maps $I$ onto $K$. Now $(g \circ f)\left(\Gamma^{n}\right)=K$ contradicting the smallness of $\Gamma$.

\section{Model THEORY OF $(\mathbb{R}, \Gamma)$}

Let $\mathbb{A}(\mathbb{R}) \subseteq \mathbb{R}^{m}$ be a one dimensional group definable in the real field $\mathbb{R}$ over $\mathbb{Q}$, and fix a subgroup $\Gamma$ of $\mathbb{A}(\mathbb{R})$ with the Mordell-Lang property such that $|\Gamma / n \Gamma|$ is finite for every $n>0$. The structure we are interested in is $(\mathbb{R}, \Gamma)$ in the language $\mathcal{L}_{\mathrm{o}}(P)$. For simplicity of notation we denote $\mathcal{L}_{\mathrm{o}}(\Gamma)$-structures by $\left(K,(\gamma)_{\gamma \in \Gamma}\right)$, rather than $\left(K,(\pi(\gamma))_{\gamma \in \Gamma}\right)$; similarly

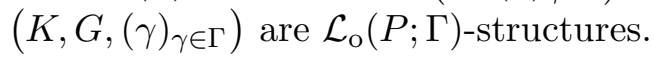

3.1. The theory. Let $T$ be $\mathcal{L}_{\mathrm{o}}(\Gamma)$-theory of $\left(\mathbb{R},(\gamma)_{\gamma \in \Gamma)}\right)$ and let $T(\Gamma)$ be the $\mathcal{L}_{\mathrm{o}}(P ; \Gamma)$-theory extending $T$ whose models are of the form $(K, G)$ satisfying the following:

(1) $G$ is a dense subgroup of $\mathbb{A}(K)$, 
(2) $\Gamma$ is a pure subgroup of $G$,

(3) for every $n>0,[n] G=[n] \Gamma$,

(4) for every $n>0,|G / n G|=|\Gamma / n \Gamma|$,

(5) $\left(K, G,(\gamma)_{\gamma \in \Gamma}\right)$ satisfies the Mordell-Lang axioms for $\Gamma$.

Note that if $\Gamma$ is dense in $\mathbb{A}(\mathbb{R})$, then $(\mathbb{R}, \Gamma)$ is a model of $T(\Gamma)$. We are proceeding to show that $T(\Gamma)$ is complete and has quantifier elimination up to formulas of the form

$$
\exists y_{1} \cdots \exists y_{m n}\left(\bigwedge_{j=0}^{n-1} P\left(y_{m j+1}, \ldots, y_{m j+m}\right) \wedge \phi\left(x, y_{1}, \ldots, y_{m n}\right)\right)
$$

where $x$ is a tuple of distinct variables and $\phi$ is a formula in the language $\mathcal{L}_{\mathrm{o}}(\Gamma)$. We achieve these by constructing a back-and-forth system between models of $T(\Gamma)$. First we prove some lemmas that will be useful in that process. In the rest of this section $(K, G)$ ranges over models of $T(\Gamma)$.

Lemma 3.1. Let $H$ be a pure subgroup of $G$ containing $\Gamma$ and $g \in G$. Then

$$
\left(\mathbb{Q}(H, g)^{\mathrm{rc}}\right)^{m} \cap G=H_{G}\langle g\rangle .
$$

Proof. It is easy to see that $H_{G}\langle g\rangle \subseteq\left(\mathbb{Q}(H, g)^{\mathrm{rc}}\right)^{m} \cap G$ since all the torsion elements of $G$ are algebraic over $\mathbb{Q}$. Now take $g^{\prime} \in\left(\mathbb{Q}(H, g)^{\mathrm{rc}}\right)^{m} \cap G$. Since $G$ and $H$ have the same Mordell-Lang solutions over $\mathbb{Q}$, we can apply Lemma 2.6 with $\mathbb{Q}$ in the place of $L$ to get that $g$ and $g^{\prime}$ are linearly dependent over $H$. Thus $g^{\prime} \in H_{G}\langle g\rangle$.

We can strengthen this lemma as follows.

Lemma 3.2. Let $H, g$ be as in the previous lemma and let $A$ be a subset of $K$ algebraically independent over $\pi(G)$. Then

$$
\left(\mathbb{Q}(A, H, g)^{\mathrm{rc}}\right)^{m} \cap G=H_{G}\langle g\rangle .
$$

Proof. By the previous lemma, all we need to show is

$$
\left(\mathbb{Q}(A, H, g)^{\mathrm{rc}}\right)^{m} \cap G \subseteq\left(\mathbb{Q}(H, g)^{\mathrm{rc}}\right)^{m} \cap G .
$$

Let $g^{\prime} \in\left(\mathbb{Q}(A, H, g)^{\mathrm{rc}}\right)^{m} \cap G$. So $\pi\left(g^{\prime}\right) \in \mathbb{Q}(A, g, H)^{\mathrm{rc}}$. Let $A^{\prime}$ be a minimal subset of $A$ such that $\pi\left(g^{\prime}\right) \in \mathbb{Q}\left(A^{\prime}, g, H\right)^{\text {rc }}$. For a contradiction, suppose that $A^{\prime}$ is nonempty and let $a \in A^{\prime}$. By minimality of $A^{\prime}$, we have that $g^{\prime} \notin\left(\mathbb{Q}\left(A^{\prime} \backslash\{a\}, H, g\right)^{\mathrm{rc}}\right)^{m}$. But then the Steinitz Exchange Principle implies that $a \in \mathbb{Q}\left(A^{\prime} \backslash\{a\}, H, g, g^{\prime}\right)^{\mathrm{rc}}$. Since $g, g^{\prime} \in G$, we get that

$$
a \in \mathbb{Q}\left(A^{\prime} \backslash\{a\}, G\right)^{\mathrm{rc}} .
$$

This contradicts with the assumption that $A$ is algebraically independent over $\pi(G)$. Hence $A^{\prime}$ is empty and $g^{\prime} \in\left(\mathbb{Q}(H, g)^{\mathrm{rc}}\right)^{m} \cap G$.

Corollary 3.3. Let $g \in G$. If $g$ is $\mathcal{L}_{\mathrm{o}}(\Gamma)$ - $\emptyset$-definable, then $g \in \Gamma$.

Proof. Using Lemma 3.1, we have $\langle\Gamma\rangle:=\Gamma\langle 0\rangle=\left(\mathbb{Q}(\Gamma)^{\mathrm{rc}}\right)^{m} \cap G$. But $\langle\Gamma\rangle=\Gamma$ as $\Gamma$ is pure in $G$. 
Lemma 3.4. Let $f: K \rightarrow K^{m-1}$ be an $\mathcal{L}_{\mathrm{o}}(\Gamma)$ - $\emptyset$-definable function and $D$ a dense subset of $G$. Then $\pi((D \backslash \Gamma) \cap \operatorname{gr}(f))$ is dense in the interior of $\pi(\mathbb{A}(K) \cap \operatorname{gr}(f))$.

Proof. First we show that $\pi((D \backslash \Gamma) \cap \operatorname{gr}(f))$ is a subset of the interior of $\pi(\mathbb{A}(K) \cap \operatorname{gr}(f))$. Since $\pi(\mathbb{A}(K) \cap \operatorname{gr}(f))$ is a semi-algebraic set, it is a finite union of open intervals and points. It is just left to show that if any of these finitely many points is in $\pi(D)$, then it is in $\pi(\Gamma)$. So suppose $\pi(g) \in \pi(D)$ is one of these points. This implies that $g$ is $\mathcal{L}_{\mathrm{o}}(\Gamma)$ - $\emptyset$-definable, since $\pi(\mathbb{A}(K) \cap \operatorname{gr}(f))$ is. Then $g \in \Gamma$ by Corollary 3.3 .

We finish the proof by showing that $\pi((D \backslash \Gamma) \cap \operatorname{gr}(f))$ is dense in the interior of $\pi(\mathbb{A}(K) \cap \operatorname{gr}(f))$. Let $I$ be any subinterval of $\pi(\mathbb{A}(K) \cap \operatorname{gr}(f))$. We just need to find $g \in D$ with $\pi(g) \in I$. By the Monotonicity Theorem, we can assume that $f$ is continuous on $I$. Hence the graph of $f$ restricted to $I$ is an open subset of $\mathbb{A}(K)$. Since $G$ is dense in $\mathbb{A}(K)$ and $D$ is dense in $G$, there is an element $g \in D$ such that $g$ is in the graph of $f$ restricted to $I$. Hence for this $g$, we have $\pi(g) \in I$.

3.2. Back-and-forth and completeness. Fix two $|\Gamma|^{+}$-saturated models $(K, G)$ and $\left(K^{\prime}, G^{\prime}\right)$ of $T(\Gamma)$, and let $\mathcal{S}$ be the collection of $\mathcal{L}_{\mathrm{o}}(P ; \Gamma)$ isomorphisms

$$
\beta:\left(\mathbb{Q}(A, H)^{\mathrm{rc}}, H\right) \rightarrow\left(\mathbb{Q}\left(A^{\prime}, H^{\prime}\right)^{\mathrm{rc}}, H^{\prime}\right)
$$

where $H$ and $H^{\prime}$ are pure subgroups of cardinality at most $|\Gamma|$ of $G$ and $G^{\prime}$ containing $\Gamma$ and $A$ and $A^{\prime}$ are finite subsets of $K$ and $K^{\prime}$ that are algebraically independent over $\mathbb{Q}(G)$ and $\mathbb{Q}\left(G^{\prime}\right)$ respectively and $\beta(A)=A^{\prime}$.

Note that by Lemma 3.2, $\left(\mathbb{Q}(A, H)^{\mathrm{rc}}, H\right)$ and $\left(\mathbb{Q}\left(A^{\prime}, H^{\prime}\right)^{\mathrm{rc}}, H^{\prime}\right)$ as above become $\mathcal{L}_{\mathrm{o}}(P ; \Gamma)$-substructures of $(K, G)$ and $\left(K^{\prime}, G^{\prime}\right)$ respectively. Moreover the map $\beta$ is a partial elementary map between the ordered fields $K$ and $K^{\prime}$ (in the language $\mathcal{L}_{\mathrm{o}}$ ).

Lemma 3.5. The collection $\mathcal{S}$ is a back-and-forth-system.

Proof. Let $\beta:\left(\mathbb{Q}(A, H)^{\mathrm{rc}}, H\right) \rightarrow\left(\mathbb{Q}\left(A^{\prime}, H^{\prime}\right)^{\mathrm{rc}}, H^{\prime}\right)$ be in $\mathcal{S}$ and $a \in K$. By symmetry it is enough to prove that there is $\tilde{\beta} \in \mathcal{S}$ such that $\tilde{\beta}$ extends $\beta$ and $a \in \operatorname{dom}(\tilde{\beta})$. We may assume that $a \notin \operatorname{dom}(\beta)$.

Case 1: $a \in \pi(G)$.

Take $b \in K^{m-1}$ such that $(a, b) \in G$. Since $G \subseteq \mathbb{A}(K)$ and $\mathbb{A}(K)$ is $\mathcal{L}_{\mathrm{O}^{-}} \emptyset$ definable of dimension 1 , there is $\mathcal{L}_{\mathrm{o}}-\emptyset$-definable function $f: K \rightarrow K^{m-1}$ such that $b=f(a)$. Let $p(x, y)$ be the $\mathcal{L}_{\mathrm{o}}(P ; \Gamma)$-type consisting of the $\mathcal{L}_{\mathrm{O}^{-}}$ type of $(a, f(a))$ over $\mathbb{Q}(A, H)^{\mathrm{rc}}$ and for every $k \in \mathbb{Z}$ and $n>0$ one of the formulas

$$
\begin{aligned}
& k(x, y) \oplus h \in n G, \\
& k(x, y) \oplus h \notin n G,
\end{aligned}
$$

depending on whether it is true in $K$ that $k(a, f(a)) \oplus h \in n G$ or not. Further let $p^{\prime}$ be type over $\mathbb{Q}\left(A^{\prime}, H\right)^{\mathrm{rc}}$ corresponding to $p$ via $\beta$. We want 
to find an $a^{\prime} \in K^{\prime}$ such that $\left(a^{\prime}, f\left(a^{\prime}\right)\right)$ realizes $p^{\prime}$. By compactness and saturation of $\left(K^{\prime}, G^{\prime}\right)$, it is enough to show that finitely many formulas of $p^{\prime}$ can be satisfied. By o-minimality of $T$, this reduces to find for every $c, d \in \mathbb{Q}(A, H)^{\mathrm{rc}}$ with $c<a<d$ and finite collection of formulas $\phi_{1}, \ldots, \phi_{n}$ of the form (3.2) or (3.3) with $(K, G) \models \bigwedge_{i=1}^{n} \phi_{i}(a, f(a))$, an $a^{\prime} \in K^{\prime}$ with

$$
\left(K^{\prime}, G^{\prime}\right) \models \beta(c)<a^{\prime}<\beta(d) \wedge \bigwedge_{i=1}^{n} \phi_{i}\left(a^{\prime}, f\left(a^{\prime}\right)\right) .
$$

By Lemma 2.1, the set

$$
Y:=\left\{g \in G^{\prime}:\left(K^{\prime}, G^{\prime}\right) \models \bigwedge_{i=1}^{n} \phi_{i}(g)\right\}
$$

is a finite union of cosets of $M G^{\prime}$ in $G^{\prime}$ for some $M \in \mathbb{N}$. Since $M G^{\prime}$ is dense in $G^{\prime}$, we have that $Y$ is dense in $G^{\prime}$ as well. By Lemma 3.4, the set $\pi((Y \backslash \Gamma) \cap \operatorname{gr}(f))$ is dense in the interior of $\pi\left(\mathbb{A}\left(K^{\prime}\right) \cap \operatorname{gr}(f)\right)$. As $\beta$ is a partial $\mathcal{L}_{\mathrm{o}}$-elementary map, $\pi(\mathbb{A}(K) \cap \operatorname{gr}(f))$ is $\mathcal{L}_{\mathrm{o}}(\Gamma)$-definable and $a$ is in it, we can assume that the interval $(c, d)$ is a subset of $\pi(\mathbb{A}(K) \cap \operatorname{gr}(f))$. Hence it follows that the interval $(\beta(c), \beta(d))$ is a subset of $\pi\left(\mathbb{A}\left(K^{\prime}\right) \cap \operatorname{gr}(f)\right)$ and that $\pi((Y \backslash \Gamma) \cap \operatorname{gr}(f)) \cap(\beta(c), \beta(d))$ is a dense subset of $(\beta(c), \beta(d))$. Now take any $a^{\prime} \in \pi((Y \backslash \Gamma) \cap \operatorname{gr}(f)) \cap(\beta(c), \beta(d))$. This $a^{\prime}$ satisfies (3.4).

It is clear that $H_{G}\langle(a, f(a))\rangle$ and $H_{G^{\prime}}^{\prime}\left\langle\left(a^{\prime}, f\left(a^{\prime}\right)\right)\right\rangle$ are pure subgroups of $G$ and $G^{\prime}$ respectively. Let $\tilde{\beta}$ be the $\mathcal{L}_{\mathrm{o}}(P ; \Gamma)$-isomorphism which extends $\beta$ to $\mathbb{Q}(A, H, a)^{\mathrm{rc}}$ and maps $a$ to $a^{\prime}$. By conditions (3.2) and (3.3), we get for every $h \in G$ that $h \in H_{G}\langle(a, f(a))\rangle$ if and only if $\tilde{\beta}(h) \in H_{G^{\prime}}^{\prime}\left\langle\left(a^{\prime}, f\left(a^{\prime}\right)\right)\right\rangle$. Hence $\tilde{\beta}$ is an isomorphism of $\left(\mathbb{Q}(A, H, a)^{\mathrm{rc}}, H_{G}\langle(a, f(a))\rangle\right)$ and $\left(\mathbb{Q}\left(A^{\prime}, H^{\prime}, a^{\prime}\right)^{\mathrm{rc}}, H_{G^{\prime}}^{\prime}\left\langle\left(a^{\prime}, f\left(a^{\prime}\right)\right)\right\rangle\right)$ and $\tilde{\beta} \in \mathcal{S}$.

Case 2: $a \in \mathbb{Q}(A, G)^{\mathrm{rc}}$.

Let $g_{1}, \ldots, g_{n} \in G$ such that $a \in \mathbb{Q}\left(A,\left\{g_{1}, \ldots, g_{n}\right\}\right)^{\mathrm{rc}}$. By applying the previous case $n$ times, we get a $\tilde{\beta} \in \mathcal{S}$ such that $g_{1}, \ldots, g_{n} \in \operatorname{dom}(\tilde{\beta})$ and $A \subseteq \operatorname{dom}(\tilde{\beta})$. Since $\operatorname{dom}(\tilde{\beta})$ is a model of $T$, we have $a \in \operatorname{dom}(\tilde{\beta})$ with $\tilde{\beta} \in \mathcal{S}$.

Case 3: $a \notin \mathbb{Q}(A, G)^{\mathrm{rc}}$.

Let $C$ be the cut of $a$ in $\mathbb{Q}(A, H)^{\mathrm{rc}}$ and let $C^{\prime}$ be the corresponding cut of $C$ under $\beta$ in $\mathbb{Q}\left(A^{\prime}, H^{\prime}\right)^{\text {rc }}$. By saturation, we can assume that there are $p, q \in K^{\prime}$ such that every element in the interval $(p, q)$ realizes the cut $C^{\prime}$. Let $d \in K^{|A|}$ be the set $A$ written as a tuple. Let $f_{1}, \ldots, f_{n}$ be $\emptyset$-definable functions in the language $\mathcal{L}_{\mathrm{o}}(\Gamma)$. By Corollary 2.11, we know that there exists $b \in(p, q)$ such that for $i=1, \ldots, n$ and every tuple $g_{1}, \ldots, g_{l}$ of elements of $G^{\prime}$

$$
f_{i}\left(g_{1}, \ldots, g_{l}, d\right) \neq b
$$

Thus by saturation, there is an $a^{\prime} \in(p, q)$ such that $a^{\prime} \notin \mathbb{Q}\left(A^{\prime}, G^{\prime}\right)^{\mathrm{rc}}$. Since $a^{\prime}$ realizes the cut $C^{\prime}$, there is an $\mathcal{L}_{\mathrm{o}}(\Gamma)$-isomorphism $\tilde{\beta}$ from $\mathbb{Q}(A, a, H)^{\mathrm{rc}}$ 
to $\mathbb{Q}\left(A^{\prime}, a^{\prime}, H^{\prime}\right)^{\mathrm{rc}}$ extending $\beta$ and sending $a$ to $a^{\prime}$. Since $a \notin \mathbb{Q}(A, G)^{\mathrm{rc}}$ and $a^{\prime} \notin \mathbb{Q}\left(A^{\prime}, G^{\prime}\right)^{\mathrm{rc}}$, we get that

$$
\mathbb{Q}(A, a, H)^{\mathrm{rc}} \cap G=H \text { and } \mathbb{Q}\left(A^{\prime}, a^{\prime}, H^{\prime}\right)^{\mathrm{rc}} \cap G^{\prime}=H^{\prime} .
$$

Since $\beta(H)=H^{\prime}$ and $\tilde{\beta}$ extends $\beta$, we get that $\tilde{\beta}$ is an $\mathcal{L}_{\mathrm{o}}(P ; \Gamma)$-isomorphism from $\left(\mathbb{Q}(A, a, H)^{\mathrm{rc}}, H\right)$ to $\left(\mathbb{Q}\left(A^{\prime}, a^{\prime}, H^{\prime}\right)^{\mathrm{rc}}, H^{\prime}\right)$ with $\tilde{\beta}(A \cup\{a\})=A^{\prime} \cup\left\{a^{\prime}\right\}$. Thus we have that $\tilde{\beta} \in \mathcal{S}$.

Now the proof of Theorem 1.2 becomes an easy consequence of this lemma.

Proof (of Theorem 1.2.) As noted above if $\Gamma$ is dense in $\mathbb{A}(\mathbb{R})$, then we have that $(\mathbb{R}, \Gamma)$ is a model of $T(\Gamma)$. So we need to show that $T(\Gamma)$ is complete. To prove this take $|\Gamma|^{+}$-saturated models $(K, G)$ and $\left(K^{\prime}, G^{\prime}\right)$ of $T(\Gamma)$, and let $\mathcal{S}$ be as above. It only remains to show that $\mathcal{S}$ is non-empty. But it is easy to see that the identity map on $\mathbb{Q}(\Gamma)^{\text {rc }}$ belongs to $\mathcal{S}$.

3.3. Quantifier elimination. Let $x=\left(x_{1}, \ldots, x_{t}\right)$ be a tuple of distinct variables. For every $\mathcal{L}_{\mathrm{o}}(\Gamma)$-formula $\phi(x)$ of the form

$$
\exists y_{1} \cdots \exists y_{m n} \bigwedge_{j=0}^{n-1} P\left(y_{m j+1}, \ldots, y_{m j+m}\right) \wedge \psi\left(x, y_{1}, \ldots, y_{m n}\right),
$$

let $P_{\phi}$ be a new relation symbol of arity $t$, and let $\mathcal{L}_{\mathrm{o}}(P ; \Gamma)^{+}$be the language $\mathcal{L}_{\mathrm{o}}(P ; \Gamma)$ together with relation symbols $P_{\phi}$ (for various $x$ ).

Let $T(\Gamma)^{+}$is the $\mathcal{L}_{\mathrm{o}}(P ; \Gamma)^{+}$-theory extending the theory $T(\Gamma)$ by an axiom:

$$
\forall x\left(P_{\phi}(x) \leftrightarrow \phi(x)\right),
$$

for each $\phi$ of the form (3.5).

With this notation in hand we are ready to prove Theorem 1.1 in the following generality.

Theorem 3.6. The theory $T(\Gamma)^{+}$has quantifier elimination.

Proof. Let $(K, G)$ and $\left(K^{\prime}, G^{\prime}\right)$ be two $|\Gamma|^{+}$-saturated models of $T^{+}$and let $\mathcal{S}$ be the back-and-forth system between $(K, G)$ and $\left(K^{\prime}, G^{\prime}\right)$ constructed above. Also take $a=\left(a_{1}, \ldots, a_{n}\right) \in K^{n}$ and $b=\left(b_{1}, \ldots, b_{n}\right) \in\left(K^{\prime}\right)^{n}$ have the same quantifier-free $\mathcal{L}_{\mathrm{o}}(P ; \Gamma)^{+}$-type. In order to prove quantifier elimination, we just need to find $\tilde{\beta} \in \mathcal{S}$ sending $a$ to $b$. Without loss of generality, we can assume that $a_{1}, \ldots, a_{r}$ are maximally algebraically independent over $\mathbb{Q}(G)$. Since $a$ and $b$ have the same $\mathcal{L}_{\mathrm{o}}(P ; \Gamma)^{+}$-type, we get that $b_{1}, \ldots, b_{r}$ are algebraically independent over $\mathbb{Q}\left(G^{\prime}\right)$. Let $\beta$ be the $\mathcal{L}_{\mathrm{o}}(\Gamma)$-isomorphism between $\mathbb{Q}\left(a_{1}, \ldots, a_{r}\right)^{\mathrm{rc}}$ and $\mathbb{Q}\left(b_{1}, \ldots, b_{r}\right)^{\mathrm{rc}}$. We will now show that $\beta$ extends to an isomorphism $\tilde{\beta}$ in the back-and-forth-system $\mathcal{S}$ sending $a$ to $b$. Let $g_{1}, \ldots, g_{l} \in G$ be such that $a_{r+1}, \ldots, a_{n}$ are in $\mathbb{Q}\left(a_{1}, \ldots, a_{r}, g_{1}, \ldots, g_{l}\right)^{\text {rc }}$. Let $p\left(x_{1}, \ldots, x_{l}\right)$ be the $\mathcal{L}_{\mathrm{o}}(P ; \Gamma)$-type consisting of the $\mathcal{L}_{\mathrm{o}}(\Gamma)$-type of $\left(g_{1}, \ldots, g_{l}\right)$ 
over $\mathbb{Q}\left(a_{1}, \ldots, a_{r}\right)^{\mathrm{rc}}$ and for every $k_{1}, \ldots, k_{l} \in \mathbb{Z}, M \in \mathbb{N}$ and $\gamma \in \Gamma$ one of the formulas

$$
\begin{aligned}
& \bigoplus_{i=1}^{l} k_{i} x_{i} \oplus \gamma \in M G, \text { or } \\
& \bigoplus_{i=1}^{l} k_{i} x_{i} \oplus \gamma \notin M G,
\end{aligned}
$$

depending on whether $\bigoplus_{i=1}^{l} k_{i} g_{i} \oplus \gamma \in M G$. Let $p^{\prime}$ be type corresponding to $p$ under $\beta$. We want to find $h_{1}, \ldots, h_{l} \in G^{\prime}$ satisfying $p^{\prime}$. By compactness and saturation of $\left(K^{\prime}, G^{\prime}\right)$, it is enough to show that every finite subset of $p^{\prime}$ can be realized. So let $\psi\left(x, b_{1}, \ldots, b_{r}\right)$ be an $\mathcal{L}_{\mathrm{o}}(\Gamma)$-formula in $p^{\prime}$ and $\chi_{1}\left(x, b_{1}, \ldots, b_{r}\right), \ldots, \chi_{t}\left(x, b_{1}, \ldots, b_{r}\right)$ be finitely many formula in $p^{\prime}$ of the (3.6) and (3.7). Put $\chi=\bigwedge_{i=1}^{t} \chi_{i}$. By Lemma 2.1, the set

$$
\left.Y:=\left\{\left(h_{1}, \ldots, h_{l}\right) \in G^{\prime l}:\left(K^{\prime}, G^{\prime}\right) \models \chi\left(h_{1}, \ldots, h_{l}, b_{1}, \ldots, b_{r}\right)\right)\right\}
$$

is a finite union of cosets of $M G^{\prime}$ in $G^{\prime}$ for some $M \in \mathbb{N}$. So the formula $\chi_{i}\left(x, b_{1}, \ldots, b_{r}\right)$ is equivalent to an $\mathcal{L}_{\mathrm{o}}(P ; \Gamma)$-formula of the form (3.5) . Hence the disjunction $\psi \wedge \chi$ is also of this form. Hence

$$
\exists y_{1} \ldots \exists y_{l} \bigwedge_{i=1}^{l} P\left(y_{i}\right) \wedge \psi\left(y_{1}, \ldots, y_{l}, b_{1}, \ldots, b_{r}\right) \wedge \chi\left(y_{1}, \ldots, y_{l}, b_{1}, \ldots, b_{r}\right)
$$

is a quantifier-free $\mathcal{L}_{\mathrm{o}}(P ; \Gamma)^{+}$-formula. Since $\left(a_{1}, \ldots, a_{r}\right)$ and $\left(b_{1}, \ldots, b_{r}\right)$ have the same quantifier-free $\mathcal{L}_{\mathrm{o}}(P ; \Gamma)^{+}$-type, the formula

$$
\exists y_{1} \ldots \exists y_{l} \bigwedge_{i=1}^{l} P\left(y_{i}\right) \wedge \psi\left(y_{1}, \ldots, y_{l}, b_{1}, \ldots, b_{r}\right) \wedge \chi\left(y_{1}, \ldots, y_{l}, b_{1}, \ldots, b_{r}\right)
$$

holds in $\left(K^{\prime}, G^{\prime}\right)$. So $p^{\prime}$ is finitely satisfiable. Now let $h_{1}, \ldots, h_{l} \in G^{\prime}$ realize $p^{\prime}$. Then $\beta$ extends to a field isomorphism

$$
\tilde{\beta}: \mathbb{Q}\left(a_{1}, \ldots, a_{r}, g_{1}, \ldots, g_{l}\right)^{\mathrm{rc}} \rightarrow \mathbb{Q}\left(b_{1}, \ldots, b_{r}, h_{1}, \ldots, h_{l}\right)^{\mathrm{rc}} .
$$

By the construction of $g_{1}, \ldots, g_{l}$ and $h_{1}, \ldots, h_{l}$, we have that

$$
\bigoplus_{i=1}^{l} k_{i} g_{i} \oplus \gamma \in M G \text { if and only if } \bigoplus_{i=1}^{l} k_{i} h_{i} \oplus \beta(\gamma) \in M G^{\prime}
$$

for all $k_{1}, \ldots, k_{l} \in \mathbb{Z}, M \in \mathbb{N}$ and $\gamma \in \Gamma$. Hence $\tilde{\beta}$ is an $\mathcal{L}_{\mathrm{o}}(P ; \Gamma)$-isomorphism of

$$
\begin{gathered}
\left(\mathbb{Q}\left(a_{1}, \ldots, a_{r}, g_{1}, \ldots, g_{l}\right)^{\mathrm{rc}}, \Gamma_{G}\left\langle g_{1}, \ldots, g_{l}\right\rangle\right) \text { and } \\
\left(\mathbb{Q}\left(b_{1}, \ldots, b_{r}, h_{1}, \ldots, h_{l}\right)^{\mathrm{rc}}, \Gamma_{G^{\prime}}\left\langle h_{1}, \ldots, h_{l}\right\rangle\right) .
\end{gathered}
$$

It is also easy to see that $\tilde{\beta} \in \mathcal{S}$, finishing the proof. 
3.4. Induced structure. Let $(K, G)$ be a model of $T(\Gamma)$. Here we study the subsets of $G^{n}$ definable in $(K, G)$.

Proposition 3.7. Let $X \subseteq G^{n}$ be definable in $\left(K, G,(\gamma)_{\gamma \in \Gamma}\right)$ with parameters from a finite set $B$. Then there is a set $E \subseteq K^{m n}$ definable in $\left(K,(\gamma)_{\gamma \in \Gamma}\right)$ over $B, \gamma_{1}, \ldots, \gamma_{l} \in \Gamma^{n}, k \in \mathbb{Z}^{n}$ and $s \in \mathbb{N}$ such that

$$
X=E \cap \bigcup_{i=1}^{l} \gamma_{i} \oplus(s G)^{n} .
$$

Proof. We may assume that $(K, G)$ is a $|\Gamma|^{+}$-saturated model of $T(\Gamma)$. Let $\mathcal{S}$ be the back and forth system of $\mathcal{L}_{\mathrm{o}}(P ; \Gamma)$-isomorphisms between $(K, G)$ and itself constructed above. Take $g, h \in G^{n}$ such that for every $E \subseteq K^{m n}$ definable in $\left(K,(\gamma)_{\gamma \in \Gamma}\right)$ over $B, \gamma_{1}, \ldots, \gamma_{l} \in \Gamma^{n}, k \in \mathbb{Z}^{n}$ and $s \in \mathbb{N}$ we have that

$$
g \in E \cap \bigcup_{i=1}^{l} \gamma_{i} \oplus(s G)^{n} \Leftrightarrow h \in E \cap \bigcup_{i=1}^{l} \gamma_{i} \oplus(s G)^{n} .
$$

Further let $C$ be a subset of $B$ which is algebraically independent over $G$. It suffices to show that there is $\beta \in \mathcal{S}$ fixing $C$ such that $\beta$ maps $g$ to $h$. Since $h$ satisfies all $\mathcal{L}_{\mathrm{o}}(\Gamma)$-formulas over $C$ which are satisfied by $g$, there is an $\mathcal{L}_{\mathrm{o}}(\Gamma)$-isomorphism from $\mathbb{Q}(g, C)^{\mathrm{rc}}$ to $\mathbb{Q}(h, C)^{\mathrm{rc}}$ fixing $C$ and mapping $g$ to $h$. We now show that $\beta \in \mathcal{S}$. It is only left to prove that $\beta(\Gamma\langle g\rangle)=\Gamma\langle h\rangle$. Since $\beta$ maps $g$ to $h$, it is enough to show for all $\gamma \in \Gamma^{n}, k \in \mathbb{Z}^{n}$ and $s \in \mathbb{N}$ that

$$
g \in \gamma \oplus D_{k, s} \text { if and only if } h \in \gamma \oplus D_{k, s} .
$$

By Lemma 2.1, there is are $\gamma_{1}, \ldots, \gamma_{l_{1}}, \delta_{1}, \ldots, \delta_{l_{2}} \in \Gamma^{n}$ such that $\gamma \oplus D_{k, s}=$ $\bigcup_{i=1}^{l} \gamma_{i} \oplus(s G)^{n}$ and $G^{n}-\left(\gamma \oplus D_{k, s}\right)=\bigcup_{i=1}^{l} \delta_{i} \oplus(s G)^{n}$. Because $h$ satisfies $p$, we have $g \in \gamma \oplus D_{k, s}$ if and only if $h \in \gamma \oplus D_{k, s}$. Hence $\beta(\Gamma\langle g\rangle)=\Gamma\langle h\rangle$ and $\beta \in \mathcal{S}$.

Corollary 3.8. Let $X \subseteq G^{n}$ be definable in $\left(K, G,(\gamma)_{\gamma \in \Gamma}\right)$ with parameters from a finite set $B$. Then the topological closure $\bar{X}$ of $X$ is definable in $\left(K,(\gamma)_{\gamma \in \Gamma)}\right.$ over $B$.

Proof. It suffices to prove that there is a set $E \subseteq K^{m n}$ definable in $\left(K,(\gamma)_{\gamma \in \Gamma}\right)$ over $B$ such that $X$ is a dense subset of $E$. We prove this statement by induction on $n$. For $n=0$, the case is trivial. So let $n>0$. By Proposition 3.7. there is an $\mathcal{L}_{\mathrm{o}}(\Gamma)$ - $B$-definable set $E_{0}$ and an $\mathcal{L}_{\mathrm{o}}(\Gamma)$ - $\emptyset$-definable set $D_{0}$ which is dense in $\Gamma$ such that $X=E_{0} \cap D_{0}$. Without loss of generality, we can assume that $E_{0} \subseteq \mathbb{A}(K)^{n}$ and that $E_{0}$ is a cell. First consider the case that $E_{0}$ is open. Then $D_{0}$ is dense $E_{0}$ and so is $X$. Now consider the case that $\operatorname{dim} E_{0}=l$ for $l<n m$. We can assume that there are an open cell $C \subset K^{l}$, a projection $\pi: K^{m n} \rightarrow K^{l}$ and an $\mathcal{L}_{\mathrm{o}}(\Gamma)$-B-definable continuous functions $f$ such that $\pi\left(E_{0}\right)=C$ and $f(C)=E_{0}$. Consider the set

$$
V^{\prime}:=\left\{\left(g_{1}, \ldots, g_{l}\right) \in \pi\left(G^{n}\right) \cap C: f\left(g_{1}, \ldots, g_{l}\right) \in G^{n}\right\} .
$$


By the induction hypothesis, there is an $\mathcal{L}_{\mathrm{o}}(\Gamma)$ - $B$-definable subset $E_{1}$ of $C$ such that $V^{\prime}$ is dense in $E_{1}$. By continuity of the $f$, the image of $V^{\prime}$ under $f$ is dense in the image of $E_{1}$ under $f$. Set $E:=f\left(E_{1}\right)$. Since $V=f\left(V^{\prime}\right)$, we have that $V$ is dense $E$.

\section{Open CORE}

In this section we prove that there are no open sets that are definable in the structures studied in the previous sections that are not definable in the field of reals. This is to say that their open core, as will be defined below, is nothing more than the field of reals. However we work in a much more general setting. So let $\mathcal{R}=(R,<,+, \ldots)$ be an o-minimal expansion of a densely ordered abelian group in a language $\mathcal{L}=\{<,+, \ldots\}$ and let $T_{\mathcal{R}}$ be its theory. Take a small subset $G$ of $R^{m}$, and let $T_{\mathcal{R}}(G)$ be the theory of $(\mathcal{R}, G)$ in the language $\mathcal{L}(P)=\mathcal{L} \cup\{P\}$, where $P$ is a new $m$-ary relation symbol. We denote models of $T_{\mathcal{R}}(G)$ by $(\mathcal{M}, P)$, where $\mathcal{M}$ is an $\mathcal{L}$-structure. Note that we do not make a distinction between the relation symbol $P$ and its interpretation. For convenience in some of the proofs we also assume that $\mathcal{L}$ has constant symbol $c$. This way we can combine two $\mathcal{L}$-definable functions by preserving the parameter set as follows: Let $f_{1}: X_{1} \rightarrow M$ and $f_{2}: X_{2} \rightarrow M$ be two functions definable in $\mathcal{M}$ over $B$, then the function $f:\left(X_{1} \times\{c\}\right) \cup\left(\{c\} \times X_{2}\right) \rightarrow M$ given by $f\left(x_{1}, c\right)=f_{1}\left(x_{1}\right)$ and $f\left(c, x_{2}\right)=f_{2}\left(x_{2}\right)$ where $x_{i} \in X_{i}$ is definable in $\mathcal{M}$ over the same parameter set $B$.

Definition 4.1. Let $\mathcal{A}=(A,<, \ldots)$ be an ordered structure and let $T$ be its theory

(i) The open core, denoted by $\mathcal{A}^{\circ}$, of $\mathcal{A}$ is the the structure $(A,(U))$, where $U$ ranges over definable open subsets of $A^{n}$ for every $n>0$.

(ii) We say that a theory $T$ is an open core of $T^{\prime}$ if for every $\mathcal{B}^{\prime} \models T^{\prime}$, there is $\mathcal{B}=T$ such that $\left(\mathcal{B}^{\prime}\right)^{\circ}$ is interdefinable with $\mathcal{B}$.

Our main result is the following.

Theorem 4.2. Suppose that for every $(\mathcal{M}, P) \models T_{\mathcal{R}}(G)$ we have that

(i) every subset of $M^{s}$ definable in $(\mathcal{M}, P)$ is a boolean combination of subsets of $M^{s}$ defined by

$$
\exists y_{1} \ldots \exists y_{m n} \bigwedge_{j=0}^{n-1} P\left(y_{m j+1}, \ldots, y_{m j+m}\right) \wedge \phi\left(x, y_{1}, \ldots, y_{m n}\right),
$$

where $\phi$ is an $\mathcal{L}$-formula, and

(ii) for every set $V \subset P^{s}$ definable in $(\mathcal{M}, P)$ over a parameter set $B$, its topological closure $\bar{V} \subseteq M^{m s}$ is definable in $\mathcal{M}$ over $B$.

Then $T_{\mathcal{R}}$ is an open core of $T_{\mathcal{R}}(G)$. 
Remark. Note that on the basis of Theorem 3.6 and Corollary 3.8, this result gives that the theory $T(\Gamma)$ as in Section 3 has o-minimal open core, namely RCF.

We prove Theorem 4.2 using the following special case (precisely when $T$ is o-minimal) of Theorem 4.14 from [3].

Theorem 4.3. Let $T$ be an o-minimal theory in a language $\mathcal{L}$, extending the theory of densely ordered abelian groups and let $T^{\prime} \supseteq T$. Then the following are equivalent:

(i) $T$ is an open core of $T^{\prime}$.

(ii) For every $\mathcal{A} \models T^{\prime}$, every unary open set definable in $\mathcal{A}$ is a finite union of intervals and every cofinitely continuous unary function definable in $\mathcal{A}$ is given piecewise by function definable in the $\mathcal{L}$-reduct of $\mathcal{A}$.

We begin with some technical results. In what follows we assume that every model $(\mathcal{M}, P)$ of $T_{\mathcal{R}}(G)$ satifies the conditions (i) and (ii) of Theorem 4.2. Note that condition (ii) is equivalent to the following condition:

(ii') For every $V \subseteq P^{s}$ definable in $(\mathcal{M}, P)$ over a parameter set $B$, there is $E \subseteq M^{m s}$ definable in $\mathcal{M}$ over $B$ such that $V$ is a dense subset of E.

Lemma 4.4. Let $X \subseteq M^{m n}$ and $f: X \rightarrow M^{k}$ be definable in $\mathcal{M}$ over a parameter set $B$ and let $V \subset P^{n}$ be definable in $(\mathcal{M}, P)$ over $B$. Then there is $E \subseteq M^{m n}$ definable in $\mathcal{M}$ such that $X \cap V$ is a dense subset of $E$ and $f(X \cap V)$ is dense in $f(E)$.

Proof. By cell decomposition in $\mathcal{M}$, we can assume that $X$ is a cell and $f$ is continuous on $X$. By $\left(\mathrm{ii}^{\prime}\right)$, there is an $\mathcal{L}$-definable set $E$ such that $X \cap V$ is dense in $E$. Since $f$ is continuous on $X$, the image of $X \cap V$ is dense in the image of $E$.

Lemma 4.5. Let $X \subseteq M^{m n}$ and $f_{1}, f_{2}: X \rightarrow M$ be definable in $\mathcal{M}$ over a parameter set $B$ and let $D \subset X \cap P^{n}$ be definable in $(\mathcal{M}, P)$ over $B$. Then the set

$$
\bigcup_{d \in D}\left\{x \in X: f_{1}(d)<x<f_{2}(d)\right\}
$$

is definable in $\mathcal{M}$ over $B$.

Proof. Let $f: X \rightarrow M^{2}$ be the function given by $x \mapsto\left(f_{1}(x), f_{2}(x)\right)$ for $x \in X$. By Lemma 4.4 there is an $\mathcal{L}$ - $B$-definable set $E$ such that $D$ is dense in $E$ and $f(D)$ is dense in $f(E)$. Hence

$$
\bigcup_{d \in D}\left\{x: f_{1}(d)<x<f_{2}(d)\right\}=\bigcup_{e \in E}\left\{x: f_{1}(e)<x<f_{2}(e)\right\} .
$$

Now note that the right hand side of the equation is $\mathcal{L}$ - $B$-definable. 
Proposition 4.6. Every unary open set definable set in $(\mathcal{M}, P)$ is definable in $\mathcal{M}$.

Proof. Let $X$ be an open subset of $M$ definable in $(\mathcal{M}, P)$ over a parameter set $B$. By condition (i) of Theorem 4.2, $X$ is a boolean combination of sets of the form

$$
\bigcup_{u \in P^{n}}\{a \in \mathcal{M}: \mathcal{M}=\phi(a, u)\}
$$

where $\phi$ is an $\mathcal{L}$ - $B$ formula. By cell decomposition, we may assume that $X$ is a boolean combination of sets of the form

$$
\begin{aligned}
& \bigcup_{u \in D} f(u)=a, \text { or } \\
& \bigcup_{u \in E} f_{1}(u)<a<f_{2}(u),
\end{aligned}
$$

where $f, f_{1}, f_{2}$ are $\mathcal{L}$ - $B$-definable functions and $D, E$ are $\mathcal{L}(P)$ - $B$-definable subset of $P^{n}$. By writing $X$ in conjunctive normal form, $X=\bigcap X_{i}$, where either $X_{i}$ or $M \backslash X_{i}$ is a finite union of sets of the form (4.2) and (4.3). Using the observation at the end of the first paragraph of this section, we may even assume that $X_{i}$ is of the form

$$
f_{1}\left(D_{1}\right) \cup\left(M \backslash f_{2}\left(D_{2}\right)\right) \cup \bigcup_{j} Y_{j} \cup\left(M \backslash Z_{j}\right),
$$

where $Y_{j}, Z_{j}$ are of the form (4.3) $, f_{1}, f_{2}$ are $\mathcal{L}$ - $B$-definable functions and $D, E$ are $\mathcal{L}(P)$ - $B$-definable subsets of $P^{n}$. Since $X$ is open, we have that $X$ is the intersection of the interiors of the $X_{i}$ 's. Hence it is only left to show that for every $i$ the interior of $X_{i}$ is $\mathcal{L}$-B-definable. So consider now the set $X_{i}$ of the form (4.4). First note that by Lemma 4.5, the set $\bigcup_{j} Y_{j} \cup\left(M \backslash Z_{j}\right)$ is $\mathcal{L}$ - $B$-definable. Thus $X_{i}$ is of the form

$$
f_{1}\left(D_{1}\right) \cup\left(M \backslash f_{2}\left(D_{2}\right)\right) \cup Y,
$$

where $Y$ is an $\mathcal{L}$ - $B$-definable set. Consider the set $f_{1}\left(D_{1}\right) \cup\left(M \backslash f_{2}\left(D_{1}\right)\right)$. By Lemma 4.4. there is an $\mathcal{L}$ - $B$-definable set $E_{1}$ such that $D_{1}$ is dense in $E_{1}$ and $f_{1}\left(D_{1}\right)$ is dense in $f\left(E_{1}\right)$. Further consider the set

$$
D:=\left\{u \in D_{2}:(\mathcal{M}, P) \models \forall v \in D_{1} f_{1}(v) \neq f_{2}(u)\right\}
$$

Again, by Lemma 4.4, there is an $\mathcal{L}$ - $B$-definable set $E_{2}$ such that $D$ is dense in $E_{2}$ and $f_{2}(D)$ is dense in $f_{2}\left(E_{2}\right)$. Hence we get by equation (4.5) that

$$
\begin{aligned}
X_{i} & =f_{1}\left(D_{1}\right) \cup\left(M \backslash f_{2}\left(D_{2}\right)\right) \cup Y \\
& =f_{1}\left(D_{1}\right) \cup\left(f_{2}\left(E_{2}\right) \backslash f_{2}(D)\right) \cup\left(M \backslash f_{2}\left(E_{2}\right)\right) \cup Y .
\end{aligned}
$$

Note that $D$ is defined in a way to guarantee that $f_{1}\left(D_{1}\right) \cup\left(f_{2}\left(E_{2}\right) \backslash f_{2}(D)\right)$ is codense in the interior of its topological closure. Set $Y^{\prime}:=\left(M \backslash f_{2}\left(E_{2}\right)\right) \cup Y$. We are going to show that the interior of $X_{i}$ is a subset of the topological closure $\overline{Y^{\prime}}$ of $Y^{\prime}$. For a contradiction, suppose $x$ is in the interior of $X_{i}$ and not in $\overline{Y^{\prime}}$. Hence there is an open interval $I \subset X_{i} \backslash \overline{Y^{\prime}}$ containing $x$. 
But $X_{i} \backslash \overline{Y^{\prime}}$ is codense in in the interior of its topological closure. This is a contradiction to $I \subset X_{i} \backslash \overline{Y^{\prime}}$. Hence the interior of $X_{i}$ is in the interior of $\overline{Y^{\prime}}$. But for every set definable in an o-minimal structure, the interior of the topological closure of a definable set is just the interior of the set itself. Hence the interior of $X_{i}$ is in the interior of $Y^{\prime}$. Since $Y^{\prime} \subseteq X_{i}$, we have that $X_{i}$ is the interior of $Y^{\prime}$ and so is $\mathcal{L}$ - $B$-definable.

Proposition 4.7. Every cofinitely continuous $\mathcal{L}(P)$-definable function is piecewise $\mathcal{L}$-definable.

Proof. Let $B \subset M$ be a parameter set and $f: M \rightarrow M$ a cofinitely continuous function which is defined by an $\mathcal{L}(P)$-B-formula $\phi(x, y)$. By Lemma 4.5 we can assume that $\phi(x, y)$ is a conjunction of disjunction of formulas of the form

(1) $\forall u \in D_{1} \quad y \neq f_{1}(x, u)$,

(2) $\exists v \in D_{2} y=f_{2}(x, v)$,

(3) $\forall w \in D_{3} x \neq f_{3}(w)$

(4) $\exists z \in D_{4} x=f_{4}(z)$,

(5) an $\mathcal{L}$ - $B$-formula $\psi(x, y)$

where $f_{1}, f_{2}, f_{3}, f_{4}$ are $\mathcal{L}$ - $B$-definable functions and $D_{1}, D_{2}, D_{3}, D_{4}$ are $\mathcal{L}(P)$ $B$-definable subsets of $P^{n}$. Let $g_{1}, \ldots, g_{k}$ be the $\mathcal{L}$ - $B$-definable functions and $C_{1}, \ldots, C_{k}$ the $\mathcal{L}(P)$ - $B$-definable subsets of $P^{n}$ in formulas of form 3 and 4 which occur in $\phi$. Since $P$ is small, the set $\bigcup_{i=1}^{k} g_{i}\left(C_{i}\right)$ is codense in $\mathcal{M}$.

Consider now $a \notin \bigcup_{i=1}^{k} g_{i}\left(C_{i}\right)$. We show that $f(a)$ is $\mathcal{L}$ - $B$-definable from $a$. By our assumption on $a$, we can assume that $\phi$ is of the the form

$$
\forall u \in D_{1} y \neq f_{1}(x, u) \wedge \exists v \in D_{2} y=f_{2}(x, v) \wedge \psi(x, y),
$$

where $f_{1}, f_{2}$ are $\mathcal{L}$ - $B$-definable functions, $D_{1}, D_{2}$ are $\mathcal{L}(P)$ - $B$-definable subsets of $P^{n}$ and $\psi$ is an $\mathcal{L}$ - $B$-formula. Set $X_{a}:=\{y: \mathcal{M} \models \psi(a, y)\}$ and define $D_{a}$ to be the set

$$
\left\{u \in D_{2}:(\mathcal{M}, P) \models \forall v \in D_{1} f_{1}(a, v) \neq f_{2}(a, v)\right\} .
$$

By Lemma 4.4 there is an $\mathcal{L}$ - $(B, a)$-definable set $E_{a}$ such that $D_{a}$ is dense in $E_{a}$ and $f_{2}\left(D_{a}\right)$ is dense in $f_{2}\left(E_{a}\right)$. First note that $f(a)$ must be in the $\mathcal{L}$ - $(B, a)$-definable set $Y_{a}:=f_{2}\left(E_{a}\right) \cap X_{a}$. If there is no open interval around $f(a)$ in $Y_{a}$, then $f(a)$ is $\mathcal{L}$ - $B$-definable over $a$. So assume for a contradiction, that there is an open interval $I$ around $f(a)$ in $Y_{a}$. Then $f_{2}\left(D_{a}\right) \cap I$ is dense in $I$. Hence there are infinitely many $b \in I$ such that $\phi(a, b)$ holds. This is contradiction to $f$ being a function. Hence $f(a)$ is $\mathcal{L}$ - $B$-definable over $a$.

By the compactness theorem, we get finitely many $\mathcal{L}$ - $B$-definable functions $h_{1}, \ldots, h_{s}$ such that for every $a \in M \backslash \bigcup_{i=1}^{k} g_{i}\left(C_{i}\right)$ there is $i \in\{1, \ldots, s\}$ with $f(a)=h_{i}(a)$. Let $Z_{0}$ be the finite set of points of discontinuity of $f$. By monotonicity theorem of o-minimal structures, there is a finite set $Z_{1}$ such that for every $c, d \in Z_{1}$ with $(c, d) \cap Z_{1}=\emptyset$ and for $i, j \in\{1, \ldots, s\}$, $h_{i}, h_{j}$ are monotone on $(c, d)$, and either $h_{i}$ and $h_{j}$ are equal on $(a, b)$ or

- $h_{i}(x)<h_{j}(x)$ for every $x \in(c, d)$ or 
- $h_{i}(x)>h_{j}(x)$ for every $x \in(c, d)$.

Hence for $c, d \in Z_{0} \cup Z_{1}$ with $(c, d) \cap Z_{0} \cup Z_{1}=\emptyset$, $f$ is continuous on $(c, d)$. Further there is dense subset $W$ of $(c, d)$ such that for every $w \in W$ $f(w)=h_{i}(w)$ for some $i \in\{1, \ldots, s\}$. Since $f$ is continuous on $(c, d)$ and all the $h_{i}$ 's are a positive distant apart, it follows from o-minimality that there is $i \in\{1, \ldots, s\}$ such that $f$ and $h_{i}$ are equal on a dense subset of $(c, d)$ and hence equal on $(c, d)$. So $f$ is given piecewise by $\mathcal{L}$ - $B$-definable functions.

Now Theorem 4.2 follows directly from Theorem 4.3 in combination with Propositions 4.6, 4.7.

Remark. As a consequence of Theorem 4.2, we take care of an unfinished business from [7]. Namely we can simplify Theorem 1.3 of that paper by removing the assumption (iii), since it follows from the first two assumptions using Theorem 4.2 .

\section{REFERENCES}

[1] Oleg Belegradek and Boris Zilber. The model theory of the field of reals with a subgroup of the unit circle. J. Lond. Math. Soc. (2), 78(3):563-579, 2008.

[2] Alexander Berenstein, Clifton Ealy, and Ayhan Günaydın. Thorn independence in the field of real numbers with a small multiplicative group. Ann. Pure Appl. Logic, 150(1-3):1-18, 2007.

[3] Alfred Dolich, Chris Miller, and Charles Steinhorn. Structures having o-minimal open core. To appear in Trans. Amer. Math. Soc.

[4] Lou van den Dries and Ayhan Günaydın. The fields of real and complex numbers with a small multiplicative group. Proc. London Math. Soc. (3), 93(1):43-81, 2006.

[5] Gerd Faltings. The general case of S. Lang's conjecture. In Barsotti Symposium in Algebraic Geometry (Abano Terme, 1991), volume 15 of Perspect. Math., pages 175182. Academic Press, San Diego, CA, 1994.

[6] Ayhan Günaydın. Model Theory of Fields with Multiplicative Groups. PhD thesis, University of Illinois at Urbana-Champaign, 2008.

[7] Ayhan Günaydın and Philipp Hieronymi. Dependent pairs. Submitted.

[8] H. Jerome Keisler. Complete theories of algebraically closed fields with distinguished subfields. Michigan Math. J., 11:71-81, 1964.

[9] Serge Lang. Number theory. III, volume 60 of Encyclopaedia of Mathematical Sciences. Springer-Verlag, Berlin, 1991. Diophantine geometry.

[10] Anand Pillay. On groups and fields definable in o-minimal structures. J. Pure Appl. Algebra, 53(3):239-255, 1988.

[11] Joseph H. Silverman. The arithmetic of elliptic curves, volume 106 of Graduate Texts in Mathematics. Springer-Verlag, New York, 1992. Corrected reprint of the 1986 original.

[12] Boris Zilber. Complex roots of unity on the real plane. Available at the webpage www.maths.ox.ac.uk/ zilber, 2003.

Fields Institute, 222 College Street, Second Floor, Toronto, ON, M5T 3J1, CANADA 
Department of Mathematics \& Statistics, McMaster University, 1280 Main Street West Hamilton, ON, L8S 4K1, Canada

E-mail address: agunaydi@fields.utoronto.ca

E-mail address: P@hieronymi.de 